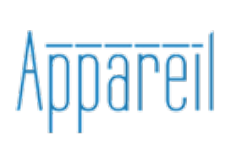

Appareil

$1 \mid 2008$

Le milieu des appareils

\title{
La destinée cybernétique de l'occident. McCulloch, Heidegger et la fin de la philosophie
}

\section{Erich Hörl}

\section{(2) OpenEdition}

1 Journals

\section{Édition électronique}

URL : http://journals.openedition.org/appareil/132

DOI : 10.4000/appareil.132

ISSN : 2101-0714

Éditeur

MSH Paris Nord

Référence électronique

Erich Hörl, «La destinée cybernétique de l'occident. McCulloch, Heidegger et la fin de la philosophie », Appareil [En ligne], 1 | 2008, mis en ligne le 17 février 2008, consulté le 30 juillet 2020. URL : http:// journals.openedition.org/appareil/132; DOI : https://doi.org/10.4000/appareil.132

Ce document a été généré automatiquement le 30 juillet 2020.

\section{(c) (i) (3)}

Appareil est mis à disposition selon les termes de la Licence Creative Commons Attribution - Pas d'Utilisation Commerciale - Pas de Modification 4.0 International. 


\title{
La destinée cybernétique de l'occident. McCulloch, Heidegger et la fin de la philosophie
}

\section{Erich Hörl}

\author{
« Notre aventure est, en effet, une grande \\ hérésie. » \\ Warren S. McCulloch \\ « Mais certains parviennent encore à percevoir \\ que penser ne signifie pas calculer. » \\ Martin Heidegger
}

\section{1.}

1 Aux yeux de James Clerk Maxwell, le lien entre les opérations mentales et les faits cérébraux ne pouvait être clarifié qu'au prix d'un certain risque métaphysique ${ }^{1}$. En tous les cas, le chemin qui menait à la région sombre et reculée où l'activité de l'esprit attendait de pouvoir être éclairée passait d'abord par la «caverne du métaphysicien ». L'espace d'un instant, lors d'une conférence prononcée devant des collègues en 1870, le mathématicien et physicien britannique sembla rêver d'une physique de la pensée mathématique, qui aurait étudié les relations entre «les opérations intellectuelles du mathématicien et le mouvement physique des molécules ${ }^{2}$ ». Mais la perspective d'avoir à traverser sur son chemin les cavernes des systèmes métaphysiques et d'y croiser les « restes des explorateurs passés » fit finalement reculer Maxwell.

2 L'effroi de ce dernier n'est pas seulement l'expression de la hantise qu'éprouve le mathématicien et scientifique à la vue des longues ombres métaphysiques qui assombrissent la question psycho-physique. Ses origines historiques et épistémologiques sont parfaitement identifiables: à l'époque de la grande transformation épistémique que constitua l'évolution de la pensée intuitive vers la pensée symbolique (dont Maxwell, le démystificateur des mondes électrophysiques, 
était d'ailleurs le protagoniste prééminent), il fallait à tout prix éviter de retomber dans les espaces de projection et les théâtres d'ombres de la pensée occidentale. Or, il était alors impossible d'appréhender la relation entre les pensées et les faits physiologiques sans recourir à des images, en se limitant à une interprétation purement symbolique de cette relation, dans le langage d'un calcul ou par le biais d'un système d'équations différentielles partielles. Il était donc fortement déconseillé à un " homme de science " d'approfondir ce problème qui visiblement ne pouvait être résolu de manière symbolique. Certes, George Boole, dans son ouvrage fondamental sur les Lois de la pensée, avait bien fait un premier pas en direction d'une calculabilité des opérations mentales, amenée à devenir l'élément central d'une nouvelle "science de l'esprit». Mais jusqu'à la fin des années 1930 et au début des années 1940, jusqu'à ce que l'émergence de la théorie de l'information physico-mathématique et son étroite alliance avec la neurophysiologie permette d'établir un parallèle exact entre les états d'une machine et les états de l'esprit, il était inconcevable que cette machine symbolique appelée " esprit » puisse être en tant que telle incorporée ou incorporable à des cerveaux et à des machines et que des calculs logiques puissent être reproductibles dans des circuits neuronaux ou électroniques. La vision fascinante décrite par Maxwell du scientifique parvenu à une connaissance de soi au sens le plus strict, capable d'identifier ses propres états mentaux successifs pendant un calcul, ne semblait quant à elle n'être rien de plus que le simple produit de sa fantaisie. Cette vision avait fait cependant irruption à un bien mauvais moment, invité fort malvenu de la noble société des physiciens et mathématiciens de la British Association. Et l'on peut mesurer à quel point le moment convenait peu en notant que Sir Charles Sherrington neurophysiologiste, prix Nobel et dualiste convaincu - se sentit obligé, dans ses Gifford Lectures de 1938, à la veille donc de l'instauration du parallélisme entre la machine et le cerveau qui allait se révéler fatal pour l'esprit, de rappeler la nature fantomatique de ce dernier :

L'esprit [...] hante notre espace de manière plus fantomatique encore qu'un fantôme. Invisible, intangible - c'est une chose sans même aucun contour ; ce n'est pas, à proprement parler, une " chose ». Il est dépourvu de confirmation sensitive, et le restera pour toujours. [...] L'esprit est $\mathrm{nu}^{3}$.

3 À la fin mars 1948, un autre neurophysiologiste, invité par le Philosophical Club de l'Université de Virginie, rendit compte lui aussi de son entrée dans la caverne de Maxwell. Mais si, dans sa communication au titre emprunté à Maxwell - «Through the den of the metaphysician" -, il balaya d'un énergique revers de main les mises en garde émises par ce dernier, et que le fantôme décrit par Sherrington ne l'épouvantait pas plus que cela, cela tenait au fait qu'il disposait d'un ensemble de calculs qui permettait de soumettre la formation de pensées dans les réseaux neuronaux à des lois mathématiques.

Les auditeurs de cette conférence n'en crurent probablement pas leurs oreilles. Celui qui s'adressait ainsi à eux présentait non seulement le noyau d'une nouvelle théorie universelle chargée de décrire les phénomènes de régulation et de transmission d'informations dans les êtres vivants et les machines; il prétendait en outre être en mesure de déterminer la caractéristique fondamentale de cet événement épistémique pour lequel le nom de "cybernétique " s'imposa dès cette année-là grâce à Norbert Wiener-, celle d'une étrange répétition de la physique et de l'épistémologie présocratique : 
Nous nous trouvons de nouveau ", affirmait-il ainsi, «dans l'une de ces périodes prodigieuses de progrès scientifique - semblable d'une certaine manière à celle des présocratiques, auxquels nous sommes redevables de la formulation claire et précise de nos problèmes physiques et, partant, épistémologiques. Quiconque a eu la chance de pouvoir écouter Wiener, von Neumann, Rosenblueth et Pitts débattre des problèmes afférents à ces machines à calculer modernes douées de savoir et de volonté aura eu l'étrange impression d'avoir assisté à un colloque antique ${ }^{4}$.

Un nom au moins manque cependant à cette liste des figures fondatrices de la cybernétique, qui insufflèrent une atmosphère présocratique à leur époque. Ce nom, c'est celui du conférencier lui-même : Warren McCulloch.

La manière dont McCulloch concevait la cybernétique - comme un retour à une interprétation présocratique du monde - était étroitement liée à la nouvelle physique de la communication. Grâce aux calculs d'informations, les faits physiques et les faits épistémologiques étaient de nouveau superposables, mais cette fois-ci de manière exacte. « Pour la première fois dans l'histoire de la science, nous savons comment nous savons et nous pouvons ainsi l'expliquer clairement» - car " nous sommes sur le point de pouvoir nous représenter l'individu connaissant comme une machine à calculer ${ }^{5} »$ : là était précisément la nouveauté, qui permettait, après un long détour de l'Histoire, de ressusciter et même de dépasser l'esprit présocratique.

7 Il n'y avait - c'était là l'essence de la « transformation techno-épistémique ${ }^{6}$ » provoquée par le nouvel espace de représentation qu'offrait le discours de l'information - aucune différence de principe entre les machines à calculer construites par l'homme et l'homme comme machine à calculer procréée. Le cerveau et la machine à calculer jouaient le rôle de modèle l'un de l'autre. McCulloch donna à son étude des machines procréées le nom d'épistémologie expérimentale ${ }^{7}$. Cette nouvelle discipline avait pour but d'étudier les calculs menant à l'acte de connaissance - et de les étudier en tant que calculs effectués à l'intérieur de l'individu connaissant lui-même. Dans un article qui fit date, publié en 1943 en collaboration avec Walter Pitts, McCulloch avait posé les bases de l'étude de la formation des concepts et des idées, d'une véritable mathématique de l'esprit chargée de formaliser, à la suite du projet booléen d'une operations research of the human mind, l'activité de l'esprit. L'hypothèse de départ qui guidait cet exposé était que «l'activité nerveuse étant régie par la loi du "tout ou rien"8 ", il était possible de formaliser les processus neuronaux et les transmissions d'informations entre neurones à l'aide des outils de la logique de l'assertion. En théorie - car McCulloch et Pitts ne travaillaient bien sûr que sur des modèles de réseaux neuronaux -, il était possible de concevoir pour toute pensée pensable le réseau neuronal capable de l'enclencher. Du point de vue de l'épistémologie expérimentale, la pensée et l'enclenchement étaient deux processus indissociables. La science de l'esprit, et avec elle l'étude de la pensée, devint ainsi une théorie du signal basée sur l'algèbre logique. Dorénavant, les ingénieurs et les mathématiciens n'allaient plus être les seuls à élaborer des circuits; les neuroscientifiques les avaient rejoints ${ }^{9}$.

8 Mais à la différence de ceux qui construisaient des machines à calculer, les chercheurs s'occupant de réseaux neuronaux étaient explicitement restés en guerre. McCulloch tout du moins, qui était non seulement scientifique, mais aussi officier et en tant que tel un acteur de la Guerre froide, se considérait encore en 1948 " confronté aux machines ennemies ", et voyait là la spécificité de son rapport quotidien aux machines procréées : «On ne m'a rien dit à leur sujet, et je suis chargé de trouver ce qu'elles sont, ce qu'elles font et comment elles le font. Ce sont des machines à calculer très complexes, 
constituées de $10^{10}$ relais. ${ }^{10}$ " La généalogie de la cybernétique est de toute évidence profondément ancrée dans la manière dont McCulloch concevait le rôle du scientifique. Celle-ci portait la marque des travaux de Wiener sur la prédictibilité statistique du comportement de vol des bombardiers ennemis, effectués afin de mieux diriger les batteries de défense antiaérienne, et que Wiener lui-même n'eut de cesse de décrire comme la scène originaire de la cybernétique. Même Heidegger alla jusqu'à noter, dans une conférence qu'il tint en 1965 au Burghölzli, que le "modèle anthropologique " conçu par la cybernétique «trouve son origine dans la cybernétique de l'artillerie antiaérienne $»^{11}$.

9 La caractérisation mathématique du réseau neuronal à l'aide d'un « calcul pour cellules nerveuses » (Calculus for Nerve Cells ${ }^{12}$ ) conduisit McCulloch à émettre des hypothèses surprenantes, mais qui en fin de compte s'inscrivaient dans la respectable tradition de la physiologie néokantienne ${ }^{13}$. Il se voyait au seuil d'une reformulation expérimentale de la philosophie transcendantale grâce à laquelle «l'objet du savoir inaccessible au savoir, la 'chose en soi', [n'allait] plus être inaccessible à l'individu connaissant ». Les mathématiques de l'esprit étaient chargées de clarifier le rôle du cerveau «dans les relations épistémiques entre nos théories et nos observations, et dans celles entre nos observations et les faits ${ }^{14}$ ». Et comme le système nerveux constituait la "machine logique par excellence ${ }^{15}$ ", le schématisme cessait de n'être qu'un art hermétique, enfoui dans les profondeurs inaccessibles de l'âme humaine. Les chambres d'opérations de l'imagination s'étaient transformées en une machine à calculer, qu'il était maintenant possible d'appréhender sans recourir à des images. Quant à l'idée de certitude, socle de toute la pensée moderne, elle n'était plus que l'effet d'une puissante " corruption d'informations " générant une forte probabilité pour que « ce qui traverse le système nerveux corresponde effectivement à des phénomènes du monde extérieur ${ }^{16}$ ». Pour la philosophie transcendantale telle que la réécrivait l'épistémologie expérimentale, l'individu connaissant était en premier lieu un réseau de $10^{10}$ relais. La « recherche du $\operatorname{logos}^{17}$ » devait donc dorénavant concentrer son attention sur les circuits neuronaux.

Selon McCulloch, l'univers de relais en lequel l'individu connaissant et le monde physique l'entourant avaient été transformés portait des caractéristiques héraclitéennes : il était sans cesse " en mouvement ", devant " traiter des informations qui lui parviennent par différents canaux, le traversent dans tous les sens et finissent par en ressortir ${ }^{18} »$. Héraclite, penseur du flux et du conflit, était le parrain idéal de cette théorie issue de la mobilisation générale des techniques de l'information lors de la Seconde Guerre mondiale. Alfred North Whitehead ne l'avait-il d'ailleurs pas déjà élevé au rang de précurseur de l'ontologie de l'événement et du procès, dont la compatibilité avec l'épistémologie cybernétique ne faisait aucun doute? Dès 1885, lorsque les membres de la société secrète des Cambridge Apostles avaient dû élire, après lecture d'un document à ce sujet, le philosophe le plus proche de la véritable nature des choses - " Democritus or Heraclitus?»-, Whitehead avait voté, comme la majorité des autres Apostles, en faveur d'Héraclite et d'une réalité continue, en transformation incessante, et contre un monde certes changeant, mais discret ${ }^{19}$. McCulloch sympathisa avec l'intuition héraclitéenne reformulée par Whitehead dans le langage des mathématiques et de la physique, de flux d'êtres et d'un ordre des choses conçu comme procès. Il considérait ainsi que l'idée d'un «éther des événements » telle que l'avait développée 
Whitehead dans son ouvrage The concept of nature (1920) « rendait compte de manière tout à fait correcte » du monde physique ${ }^{20}$.

11 De l'autre côté de l'Atlantique, et pour ainsi dire en guise de réponse européenne à la provocation cybernétique, un séminaire sur Héraclite débuta au semestre d'hiver 1966/1967 par une lecture attentive des fragments 64 et 41 - les passages abordant précisément les questions présocratiques de commande et non de flux ${ }^{21}$. Pour Martin Heidegger, qui mena en collaboration avec son successeur Eugen Fink cette expérimentation d'un diagnostic de son époque par le biais d'un dialogue avec le philosophe éphésien, « le sens de la cybernétique » semblait résider dans le fait qu'elle était issue de la conception héraclitéenne du phénomène de commande ${ }^{22}$. C'est pour " éclaircir ${ }^{23}$ " ce phénomène qui à ses yeux déterminait à la fois "les sciences de la nature et le comportement de l'homme " et constituait le symbole épistémique de l'époque, que fut entreprise cette interprétation d'Héraclite à la lumière de la cybernétique. Car pour parvenir à comprendre ce que commander veut dire Heidegger était sur ce point lucide -, "nous n'avons pas besoin d'une quelconque philosophie de la nature. Il suffit de bien se rendre compte d'où vient la cybernétique et où elle conduit ${ }^{24}$."

La question de la commande, qui se trouva ainsi placée au centre des préoccupations philosophiques, semblait non seulement confronter la cybernétique aux fondements métaphysiques de sa condition technologique, elle semblait aussi dévoiler son historicité, ou plutôt la base et le sens de l'histoire occidentale dans son entier. Dans un contexte où la cybernétique portait exclusivement son attention sur l'acte de commande, tout le passé se révélait être le résultat d'une commande spécifique, celle d'une orientation techno-logique initiale et de son maintien ferme, qui aurait trouvé son aboutissement dans la métaphysique cybernétique de l'acte de commande. L'ère du commander marquait cet instant où la «destinée » métacybernétique de l'Occident parvenait pour ainsi dire à soi. Ce n'est qu'ainsi que l'on peut comprendre la question formulée par Heidegger: "La cybernétique actuelle n'est-elle pas elle-même commandée ?25" Après avoir défini la faculté cybernétique de commande, en s'appuyant sur l'activité cybernétique grecque prototypique (le commandement d'un navire), comme le maintien par la force d'une orientation particulière, Heidegger insista sur un autre type de commande, plus originel et non-violent, et qui régulait à ses yeux toute l'histoire de l'Être: "Commander», dit-il, "peut être d'un côté maintenir de force dans la direction, mais d'un autre côté aussi le commandement nonviolent des Dieux ${ }^{26} »$. La question de la commande - et notamment la question de la force non-violente d'un commandement originel, qui précéderait tout commandement par la force - était maintenant très proche de ce qu'il nommait « destiner » (schicken) et la « destinée » (Geschick), et qui était censé commander jusqu'à la cybernétique ellemême, l'avoir rendue possible par l'orientation techno-logique prise initialement et maintenue par la métaphysique occidentale, puis l'avoir préparée et l'avoir faite apparaître. La destinée s'avéra cependant être un modèle de pensée protocybernétique fortement problématique, voire même dès l'abord lui-même contaminé par la technologie.

13 Mais McCulloch avait trouvé encore d'autres précurseurs à l'atmosphère présocratique qui régnait sur son époque. La machine à calculer moderne et le processeur appelé " cerveau » constituaient également de véritables appareils démocritiens, dès lors qu'ils travaillaient à l'aide d'unités discontinues infiniment petites, les « signaux quantifiés ", 
qui pouvaient ou non apparaître. "Toute machine à calculer moderne un tant soit peu effective ", affirmait-il ainsi, «travaille selon des principes démocritiens ${ }^{27}$ ». Cela était tout aussi vrai du système nerveux, dont la structure faite de neurones et l'effet mesurable en signaux et impulsions infiniment petits étaient quantifiables.

Et bien qu'il n'ait été qu'une seule fois question de «l'unité chez Parménide » - et cela encore que de manière très évasive -, c'était bien là le signe décisif d'un renouveau de la philosophie présocratique. Ne fallait-il pas voir dans la communication telle qu'elle se produisait dans l'homme et la machine la preuve de la véracité du mot de Parménide : "Car le même est à la fois penser et être ${ }^{28}$ » ? Ce mot avait en vérité tout pour devenir la devise de toute épistémologie expérimentale.

phe de Parménide constituait même la «maxime de la philosophie occidentale », dans la mesure où il «ne pouvait plus être compris, n'ayant pu maintenir sa vérité originelle». S'il fallait voir en la «chute hors de la vérité de ce $\operatorname{mot}^{29}$ » l'origine de la philosophie, dont l'histoire elle-même ne constituait « au fond [qu']une suite de variations sur ce seul thème ${ }^{30} "$, alors McCulloch, en évoquant Parménide, avait placé le programme de l'épistémologie expérimentale au cœur de la tradition occidentale. Et ce - nous le verrons bientôt - comme reprise et fin de la philosophie.

16 Cette étroite proximité de la cybernétique et de la philosophie présocratique appartient à l'histoire de la fascination que toutes deux ont exercée, et donc, d'une certaine manière, à la logique même de l'épistémè. Hermann Diels avait édité les Fragments des présocratiques en 1903. Pour la première fois, ceux-ci étaient apparus délivrés du contexte foisonnant dans lequel ils avaient été transmis et étaient devenus accessibles en tant que textes sui generis. Jusqu'à l'avènement de "l'ère dielsienne ", ils avaient été presque enfouis sous l'« épaisse couche de leur exégèse ${ }^{31}$ ». L'accessibilité retrouvée de ces textes tombait dans un contexte de fascination pour l'ancien, l'archaïque, le primitif, qui était petit à petit devenue, depuis les années 1870, la marque épistémique de l'époque. Face à la crise de la pensée catégoriale traditionnelle causée par l'apparition de l'électrophysique, des mathématiques pures, de la logistique et des moyens de communication techniques, des socioanthropologues, des ethnologues, des philosophes et des sociologues, et notamment ceux de l'école de Durkheim, s'étaient mis à la recherche des catégories primitives, préaristotéliciennes. Marcel Mauss a donné l'expression la plus précise de cette volonté de descendre jusqu'aux souterrains archaïques de la pensée :

Les catégories aristotéliciennes ne sont en effet pas les seules qui existent dans notre esprit, ou qui ont existé dans l'esprit et dont il faille traiter. Il faut avant tout dresser le catalogue le plus grand possible de catégories; il faut partir de toutes celles dont on peut savoir que les hommes se sont servis. On verra alors qu'il y a eu et qu'il y a encore bien des lunes mortes, ou pâles, ou obscures, au firmament de la raison ${ }^{32}$.

17 L'ère post-alphabétique se mit à imaginer les fondements pré-alphabétiques de la pensée afin de surmonter ses propres incertitudes épistémologiques et ontologiques. En quelque sorte, elle s'inventa elle-même dans son esquisse d'une pensée préalphabétique. Dans les modèles de pensée que la mentalité primitive avait développés resurgissaient en effet les traits d'une épistémè déterritorialisée par l'électrophysique et la logique symbolique. Martin Heidegger lui-même fut obligé d'insister sur le fait que sa lecture d'un penseur originel ne participait en aucune manière de l'illusion archaïsante d'une mentalité primitive ${ }^{33}$. La cybernétique et la théorie de l'information avaient 
conduit à un changement d'époque épistémologique par leur consolidation commune, au terme d'une longue période de gestation, d'une pensée radicale menée par le concept de fonction; il était donc en un certain sens normal de les relier à la pensée originelle. Pensée première et pensée nouvelle avaient été séparées par la longue période de l'aventure métaphysique, guidée quant à elle par le concept de substance. Cette dernière période apparaissait comme un long détour de l'Histoire, tandis que l'Histoire elle-même, purifiée scientifiquement, pouvait désormais revenir à ses commencements. McCulloch ne fut pas le seul à établir cette corrélation. Claude LéviStrauss par exemple, engagé lui aussi dans l'entreprise de cybernétisation des sciences humaines à travers son projet d'Anthropologie structurale, vit dans la cybernétique une réhabilitation fondamentale de la pensée sauvage, et dans le penseur sauvage un protocybernéticien et un théoricien de l'information avant la lettre ${ }^{34}$. De telles hypostases historiques doivent quant à elles être lues comme le symptôme de l'illusion cybernétique ${ }^{35}$ qui consista à voir en la cybernétique le facteur majeur de la crise de l'esprit occidental d'une part, et d'autre part une césure ontologique et épistémologique fondamentale, comparable uniquement avec un événement tel que l'apparition de la pensée grecque, c'est-à-dire avec la naissance de la philosophie.

Mais le court-circuit opéré par McCulloch pour joindre philosophie présocratique et cybernétique avait des causes plus précises encore. Dans sa réflexion ontologique, McCulloch avait développé une vision de la fin de la philosophie par la cybernétique, ce qui avait suffit à rapprocher cette dernière des présocratiques. Selon le compte-rendu qu'a fait Gotthard Günther de ses rencontres avec McCulloch, qui eurent lieu pendant presque toute la décennie des années 1960, celui-ci déplorait l'« absence de réflexion ontologique fondamentale ${ }^{36} »$ dont témoignaient les théoriciens de la cybernétique. Ces derniers se considéraient en effet à l'avant-garde de la pensée symbolique, et de ce fait exempts de tout questionnement ontologique. Ils étaient pour ainsi dire entrés dans cette caverne behavioriste, pourtant interdite d'accès, qu'était la "black box». Or, il était établi qu'il suffisait de se poster à ses entrées et ses sorties, et de formaliser. Pour McCulloch au contraire, toute logique et tout calcul imaginables constituaient une formalisation plus ou moins exacte de concepts ontologiques. Les réflexions ontologiques que McCulloch ne cessa de mener, et qui le poussèrent à exiger une fondation philosophique de la cybernétique, avaient une origine bien précise, qui jette une lumière particulière sur le programme cybernétique dans son ensemble. "La révélation, raconte Günther, vint un soir, lorsque McCulloch commença à parler de Martin Heidegger et alla chercher une édition de Être et temps complètement usée, presque réduite en lambeaux du fait d'une utilisation intensive." Aux yeux de McCulloch, - ces yeux qu'avait manifestement ouverts le livre de Heidegger (un cadeau de son ami Eilhard von Domarus, qui avait été l'auditeur de Husserl et de Heidegger) si la philosophie depuis Platon et Aristote était plongée dans un oubli de l'être, c'est qu'elle n'avait pas assez pris au sérieux les problèmes posés par la cybernétique. Elle avait qualifié « à tort d"être' la simple objectité sans autoréférence ", sans comprendre que l'être est «toujours à la fois sujet et objet ». L'oubli de l'être par la philosophie fut donc interprété comme un oubli initial de la cybernétique, ou plutôt comme un déficit catégorial constitutif de la philosophie, comme son incapacité à penser l'être autrement qu'à l'aide de ses seules distinctions classiques et solidement installées. La pensée cybernétique devait au contraire - c'est précisément ce qu'elle avait de commun avec les présocratiques et rendait ceux-ci déchiffrables, étant leur répétition - toujours 
déjà opérer avant ce régime différentiel classique. Comprise ainsi, elle signifiait la sortie de l'oubli de l'être, et la fin de la philosophie.

Même dotées d'une certaine profondeur historique par leur utilisation de l'idée heideggérienne d'oubli de l'être, les réflexions ontologiques menées par McCulloch restaient très en deçà de tout ce qui avait alors cours sous ce nom dans la philosophie continentale. Elles ne thématisaient ni le problème de l'objectité, ni celui de l'être de l'étant comme version finale et comme limite de toute ontologie de l'objet, naviguant au contraire dans les eaux du courant américain de l'ontologie du procès. L'être devant être pensé comme procès, comme le soutenait Whitehead, il n'y avait aucune raison de craindre à la manière des ontologues européens la dissolution de la catégorie d'objet, et avec elle l'avènement de symbolismes purement abstraits ${ }^{37}$. L'ontologie du procès permettait au contraire d'envisager un enrichissement ontologique de la cybernétique ; elle évitait ainsi de se restreindre à la seule démonstration de l'oubli irrémédiable de l'être, culminant dans son enfermement en un circuit de régulation, et donc à la tâche nécessaire - ainsi que Heidegger le laissait entendre - de sa destruction ${ }^{38}$.

Si l'on en croit Günther, la nuit devait déjà être bien profonde avant que McCulloch prononce de tels propos. Or comme chacun sait, la nuit est la mère de l'ontologie. Celui qui n'avait pas la chance de passer la nuit avec McCulloch pouvait bien sûr se contenter d'interpréter sa conférence de Virginie sur le retour de la philosophie présocratique comme l'oraison funèbre prononcée par la cybernétique sur la tombe de la philosophie. Et ce notamment en la lisant à partir de sa conclusion, qui donnait un nom à quelques ossements de la caverne décrite par Maxwell :

Voilà l'un d'eux ; il s'agit sûrement du fémur d'Emmanuel Kant - sa confusion entre l' ego empirique et l'ego épistémologique. Il maintint Kant fermement sur le sol des sciences naturelles, tandis que son crâne s'élevait vers le royaume de la théorie. En voilà un autre - son crâne, sûrement, qui abrita sa machine à calculer, le réseau de ses relais constituant son "a priori synthétique".

Et pour le cas où, contre toute attente, McCulloch ne serait pas parvenu à ressortir sain et sauf de la caverne de la métaphysique, et qu'il aurait trouvé là, aux côtés d'autres philosophes, non la fin de la philosophie, mais sa propre fin, il s'était réservé une dernière demeure aux côtés de Kant: «Si mes os devaient tomber à côté des siens, j'espère que les générations futures sauront reconnaître là ma colonne vertébrale ${ }^{39}$. »

\section{2.}

L'affaire de la pensée tel que l'épistémologie expérimentale de McCulloch le concevait constituait bien plus qu'un simple phénomène marginal : il était au centre de cette expérimentation épistémologique que fut la cybernétique. L'alliance entre l'ingénierie et la neurologie était sans aucun doute un élément central de son programme et de la conception de la pensée qui le sous-tendait. Le 24 janvier 1945, Wiener rendit compte à Arturo Rosenblueth d'une rencontre informelle tenue dans le but de définir les contours de ce nouveau champ de recherche qui ne s'appelait pas encore "cybernétique ", et dont la tâche serait de faire du savoir acquis pendant la guerre dans le domaine des systèmes de communication un savoir également utilisable en temps de paix.

Le premier jour, von Neumann parla de machines à calculer et moi d'ingénierie de

la communication. Le deuxième jour, Lorente de Nó et McCulloch firent en commun 
une présentation très convaincante de l'état actuel du problème de l'organisation du cerveau. À la fin, nous étions tous convaincus du fait que les sujets portant sur l'ingénierie et sur la neurologie ne font qu'un, et que nous devrions réfléchir à créer un projet de recherche permanent dans lequel nous pourrions développer ces idées. [...] nous avons la ferme intention de créer une société et une revue après la guerre, et de fonder à la Tech ou autre part dans le pays un centre de recherches consacré à notre nouveau champ d'études ${ }^{40}$.

Pour Wiener, le concept de " cybernétique », chargé de caractériser ce nouveau champ de recherches, devait unir « sous une seule et même appellation [...] ce qui, au sujet de l'homme, est parfois appelé de manière imprécise 'penser', et qui est connu dans le domaine de la technique sous le nom de commande et de communication ». Le but était de " développer une théorie qui recouvrirait l'ensemble des phénomènes de commande et de communication dans les machines et dans les organismes vivants ", sur la base de la détermination d'«éléments communs dans le fonctionnement des machines automatiques et $\mathrm{du}$ système nerveux ${ }^{41} »$. Même John von Neumann, qui avait certainement un bon nombre de réserves bien fondées à l'égard des tendances à la généralisation dont faisait preuve Wiener, misait sur la construction de machines à calculer chargées d'imiter le cerveau humain ${ }^{42}$. L'une des conclusions de son First Draft of a Report on the EDVAC de 1945 préconisait justement de poursuivre les travaux menés par Pitts et McCulloch sur les calculs en imitant à l'aide de tube à vide des fonctions neuronales simplifiées. Et s'il était urgent d'élaborer une nouvelle théorie logique afin de mieux comprendre le fonctionnement d'automates extrêmement complexes, et notamment celui du système nerveux central, cela ne pouvait être réalisé qu'à condition que « la logique [...] s'adapte à la neurologie, et non l'inverse, et ce par une pseudomorphose bien plus importante que toutes celles opérées jusqu'à maintenant ${ }^{43}$. " La nouvelle machine transclassique, qui devait fonctionner non plus en analogie avec la main, comme c'était le cas de la machine mécanique classique, mais avec le cerveau, devint une véritable "prothèse de pensée ${ }^{44}$ » (G. Günther) pour les recherches menées sur l'acte de penser et, plus généralement, pour la conception d'une théorie nonclassique de la pensée.

Ce fut cette expérimentation à grande échelle menée sur l'« affaire de la pensée » qui souffla à Martin Heidegger - qui après la guerre avait dans un premier temps, et pour les raisons que l'on sait, adopté la posture d'un observateur silencieux - sa question maîtresse : «Qu'est-ce que penser ? ». Ce n'est qu'à la lumière de la question de savoir si les machines pouvaient penser - question que l'époque entière se posa sous toutes les formes possibles et imaginables - et confronté à la conception technologique de la pensée, qui motivait ce questionnement, que Heidegger parvint à formuler le diagnostic principal de sa philosophie d'après-guerre : «Ce qui donne le plus à penser dans notre temps qui donne à penser est que nous ne pensons pas encore ${ }^{45}$. " À l'éradication par la cybernétique de l'inexactitude de la pensée répondit depuis Fribourg l'appel à une reformulation de la tâche de la pensée. Pour Heidegger, la «question fondamentale de l'histoire du monde ${ }^{46} »$ se concentrait sur ce point. Vinrent ensuite de longues années d'articulation de la «question de la pensée, dont la portée est universelle ${ }^{47}$ ». Il en ressortit que la pensée était ce que l'époque des machines à penser n'avait jusqu'alors et depuis bien longtemps pas encore pensé.

L'analyse noologique de l'évènement cybernétique laissa apparaitre l'attention portée presque exclusivement à la logique et aux mathématiques, qui selon Heidegger caractérisait l'Occident et dont l'analyse constitua le programme de sa vie, comme la 
condition techno-logique de possibilité de la pensée dans son ensemble. La cybernétique fit irruption dans la pensée de Heidegger avec une force telle que Derrida fut amené à dire ses mots qu'il nous reste encore à comprendre: «Il a parlé de cybernétique toute sa vie ${ }^{48}$.» En même temps, le tracé des contours de ce point aveugle de l'épistémè contemporaine permit à Heidegger, constamment soucieux d'asseoir son influence, mais politiquement désavoué, d'installer son programme dans le paysage discursif nouveau qu'offrait la Guerre froide. En travaillant à un scénario-catastrophe, applicable aussi bien au présent qu'au futur, d'un monde où tout pourrait être commandé et planifié, et en parvenant à capitaliser philosophiquement les menaces imminentes, Heidegger pouvait éviter de parler du passét9.

Le premier cours qu'il tint après la levée de son interdiction d'enseigner, intitulé Qu'estce que penser? (1951/1952), ne faisait aucun mystère de ce qui obligeait à poser cette question. La logistique - Heidegger reprenait là le terme mis en vigueur vers 1900 par Louis Couturat et Gregorius Itelson pour désigner la logique symbolique - commençait alors, "en Amérique et ailleurs, à établir son emprise sur l'esprit, comme étant la véritable philosophie de l'avenir ${ }^{50} »$. Cela avait, selon Heidegger tout du moins, des causes archéologiques parfaitement identifiables: la pensée ayant été perçue à ses débuts comme lógos, et cette interprétation masquant depuis lors la pensée, «la logistique [devenait] la forme d'organisation universelle de toute représentation ${ }^{51}$ ». À l'inverse, "[n]ous contemporains », " nous ne pouvons apprendre la pensée que si nous désapprenons radicalement son essence traditionnelle ${ }^{52}$.»

Heidegger se trouvait dès ses tout premiers travaux sur la voie d'un tel déroulement archéologique de la question logique. Son compte-rendu de «Recherches récentes sur la logique » de 1912, encore très marqué par le néokantisme et rédigé à l'occasion de la "querelle des principes », laisse apparaître une première fois la question qu'il allait poser durant toute sa vie: "Qu'est-ce que la logique? Nous sommes là devant un

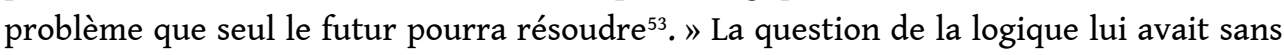
nul doute été dictée par la situation épistémique. Elle constituait en effet autour de 1900 la question directrice de l'épistémè, divisant les esprits selon qu'ils soutenaient la thèse d'un socle intuitif et concret de la pensée ou qu'ils s'en détachaient, optant dans ce cas, en conformité avec l'axiomatique naissante, pour sa pure et simple calculabilité. Ainsi Husserl, qui venait des mathématiques, avait mobilisé la phénoménologie - il convient d'ajouter: dans un acte très puissant d'auto-affirmation philosophique contre le «cliquetis » des machines symboliques, qu'un esprit intuitif ne pouvait selon lui trouver qu'absurde, et s'était fixé comme objectif de refonder la pensée intuitive par le biais d'une philosophie de la conscience interprétant les purs symbolismes, non visualisables, comme des formations de sens ${ }^{54}$.

Dans Être et temps, Heidegger contourna le mode traditionnel de questionnement des fondements, chargé d'amener une époque en crise à la conscience de soi ontologique et épistémique, et adopta le point de départ plus originel de l'analytique existentiale, se distançant ainsi de sa socialisation néokantienne. Le réexamen par l'herméneutique du Dasein du problème du jugement, c'est-à-dire de l'assertion, qui retirait au «pur intuitionner» sa "primauté » et tentait de faire apparaître l'«intuition» et la "pensée » comme des «dérivations (...) éloignées » du «comprendre " existential, restait tout à fait dans le cadre de la problématique de la logique ${ }^{55}$. L'« analyse de l'assertion » revêtait dans ce contexte une importance toute particulière. Il lui revenait

une place insigne à l'intérieur de la problématique de l'ontologie fondamentale parce que, dans les commencements décisifs de l'ontologie antique, le lógos a[vait] 
joué le rôle d'unique fil conducteur donnant accès à l'étant véritable et permettant de déterminer l'être de cet étant ${ }^{56}$. cette longue durée de prédominance du lógos était depuis bien longtemps sur le point d'être à la fois parachevée et remplacée par la logistique. La puissance avec laquelle le symbolisme transformait les fondements du savoir menait à la dissolution du jugement en un "système de 'coordinations' ", le métamorphosant en "l'objet d'un 'calcul' ", mais non en un "thème d'interprétation ontologique ${ }^{57}$ ». En faisant de la pensée un calcul - cette opposition entre la pensée et le mathem constitue certainement chez Heidegger un lourd héritage hégélien -, et plus précisément, mais cela ne deviendra évident que longtemps après Être et temps, en la transformant en une affaire aveugle et sourde aux exigences du dévoilé (Unverborgenen) et du révélé (offenbaren), la logistique devenait le haut lieu de l'oubli de l'être. C'était elle qui, par son développement d'une méthode de calcul de l'assertion, incitait comme nulle autre à poser de nouveau la question du sens de "être ». Et l'on peut même lire au paragraphe 3 de Être et temps, dans le contexte de l'« exposition de la question du sens de 'être' », que la question de l'être elle-même devait son dévoilement aux secousses épistémiques qui avaient ébranlé les fondements de la logique et des mathématiques en général.

L'importance de la question de la logique, la place centrale qu'elle occupe dans le programme heideggérien apparaissent clairement si l'on note que Heidegger ne cessa plus à partir de ce moment là de tenir des cours de logique, sous diverses appellations. On pourrait appeler cet ensemble une archéologie philosophique de la formalisation. Il aura finalement dévoilé les attitudes fondamentales de l'esprit occidental qui ont conduit depuis l'Antiquité grecque à la machinisation de la pensée et à la radicalisation progressive de la condition techno-logique. Le chemin que Heidegger dut pour cela parcourir entraîna l'intégration à son parcours théorique de la problématique des fondements et conduisit pour finir à la destruction historiquement nécessaire de sa propre discipline.

En 1928, alors qu'il enseignait à Marbourg, il évoqua d'abord une "critique de la logique traditionnelle par le dévoilement de ses fondements ${ }^{58}$ ». Le " préjugé » « selon lequel la logique ne serait rattachée à aucun socle concret, se suffisant à elle-même» voilà ce qu'il fallait "détruire ${ }^{59}$ ». Heidegger s'attaquait ainsi à l'autonomie de la logique, qu'avaient péniblement érigée les mathématiciens et les fondateurs de la logique symboliques, et, du même coup, au socle sur lequel reposait le symbolisme. Cette attention portée sur le préjugé logique était quant à elle la conséquence d'un constat, celui de la force de frappe archéologique d'une différence autour de laquelle allait désormais s'organiser la conception de l'histoire de l'être comme histoire des configurations fondamentales successives de cette différence : celle entre être et penser. C'était là la machine diagnostique de Heidegger, à l'aide de laquelle il allait pouvoir constituer et distinguer différentes époques en fonction de l'interprétation de l'étant transparaissant dans chacune de leurs manifestations respectives. C'est à travers son expression moderne radicale que cette différence lui était apparue dans toute sa splendeur historique et, de là, comme une différence archéologique fondamentale : si l'« être n'apparaît qu'après la pensée ", avait-il alors affirmé, « alors l'étant doit être en tous points tel qu'il peut être caractérisé dans son entier par la pensée ${ }^{60}$ ». Libérer la logique de sa « liaison » originelle, grecque, à l'être - telle était la « tâche » majeure que 
semblait s'être assignée la modernité, et dont la réalisation aboutissait à la disparition totale de la question de l'être dans de purs formalismes.

C'est au semestre d'été 1935 que Heidegger accomplit la percée vers le grand modèle de pensée de son archéologie, que son texte sur Le Temps de l'image du monde allait rendre célèbre : celui de la " pensée de la représentation ». Cette idée permettait de préciser la situation moderne : la "souveraineté normative » de la différence entre être et penser devenait visible là où la pensée s'opposait à l'être, de sorte que «celui-ci lui est présenté, et par suite lui fait face à la manière d'un ob-jet ». La pensée devint ainsi la base et le pôle « à partir desquels se décide le sort de ce qui lui fait face, et cela au point que l'être même reçoit son sens à partir de la pensée ${ }^{61}$ \%. Ce pouvoir d'interprétation constitua le présupposé direct à l'élaboration des calculs logiques qui, finalement, n'avaient pour but ni de présenter ni de représenter l'étant, mais de le générer comme l'effet de symbolismes, et de dissoudre ainsi toute objectité. La configuration fondamentale moderne de la différence entre penser et être portait donc déjà en elle, comme sa possibilité la plus propre, son propre dépassement vers l'imprésentable et l'irreprésentable dans lesquels s'aventurèrent les symbolismes, eux-mêmes issus de cette configuration. Le temps de l'image du monde trouva dans la représentabilité sa définition et sa limite. Dans sa propre recherche de la "parole de la pensée ", cette parole "pauvre en images ${ }^{62}$ ", dans sa recherche de l'« 'être' sans image » de la vérité, de cet «être sans image ni représentation " qui "donne à toute chose représentable son fondement et sa nécessité ${ }^{63}$ ", Heidegger - assez proche en cela, il convient de le noter, de la pensée aveugle des symbolismes purement abstraits - situa sa propre pensée à l'extrême marge de l'horizon conceptuel qu'offrait la modernité. La limite de l'ontologie traditionnelle - la désintégration de la catégorie de l'objet et l'apparition de l'imprésentable et de l'irreprésentable - fut soumise à une réinterprétation, et devint le lieu véritable de la réalisation de la vérité et de l'être. De ce point de vue, le travail de destruction effectué par Heidegger réitérait d'une manière toute particulière la destruction de l'ontologie à laquelle oeuvraient depuis la fin du XIX siècle les sciences naturelles et les mathématiques.

La différence entre être et penser fut élevée au rang de condition historique de possibilité de ce qui depuis la nuit des temps portait le nom de «logique». Cette différence en effet devait être toujours déjà réalisée avant que la logique, en tant que ce qui interprète la pensée comme ce qui a pouvoir de désigner l'être, puisse apparaître. Il fallut cependant attendre la radicalisation logistique de la cybernétique pour que Heidegger accomplisse un réel questionnement en retour de la réalisation de cette différence, puisque ce n'est qu'à la lumière de cette radicalisation qu'il parvint à percevoir toute l'historicité de cette entreprise. Heidegger ne soumit pour cela à sa réinterprétation rien d'autre que le fragment de Parménide grâce auquel McCulloch, le cybernéticien ontologue, était auparavant parvenu à se situer de manière adéquate dans l'histoire de la pensée. Plus question désormais d'une identité entre penser et être qui aurait trouvé là son inscription. En dernier lieu, le lógos, dont l'interprétation dans le sens d'une assertion avait donné à la philosophie, sans que celle-ci puisse s'en rendre compte, son orientation logique, allait ravir, charmer, captiver, se contentant de faire apparaître, de faire transparaître en rassemblant - aimylioi lógoi, comme le dit une seule fois Homère, parole douce et ravissante ${ }^{64}$. Telle était la limite initiale de toute commande et de toute prédiction - de tout pensable, même, dans sa globalité. 

démission du poste de recteur de l'Université de Fribourg, et dans il transforma la question de la logique en une question sur l'essence du langage, constitue la scène-clé de son archéologie philosophique. Car quiconque voulait «ébranler la logique depuis ses débuts et en ses fondements ${ }^{65}$ » devait tout d'abord, comme l'affirmait son programme, attaquer le primat de l'assertion, et ce non seulement par l'ontologie fondamentale, mais surtout par la réfutation de l'idée communément admise, et que ce primat transportait, selon laquelle le langage est présentation et représentation, expression et communication. La destruction de l'idée d'assertion permettait d'ôter tout fondement aux tentatives de formalisation. L'attention nouvelle portée sur les questions de langage devait contribuer à répondre à la question de la logique, ellemême étant avant tout - et à plus juste titre encore pour un professeur d'université un « service rendu à la pensée ». En tout dernier lieu, dans la dernière ligne droite de ce travail de destruction, devaient se révéler dans le voilement historique de l'être du langage, conçu comme "simple attribution de signes ", non seulement la condition de possibilité de sa «technicisation par le biais de la théorie de l'information ${ }^{66}$ », mais aussi et surtout celle de la théorie de l'information elle-même et de sa machinisation. $\mathrm{Au}$ premier abord, la "redéfinition du langage comme simple information" ne trahissait que « la spécificité de la technique moderne ${ }^{67}$ ». Mais « le lieu et la possibilité d'une refonte du langage en langage technique, c'est-à-dire en de l'information ", provenaient en fait de "l'être du langage » lui-même, comme l'affirmait Heidegger au Château de Combourg, à Schwäbisch Hall, en 1962. "Le dire comme monstration ", ainsi qu'il définissait l'être du langage, pouvait « également être présenté et réalisé de telle façon que montrer signifie seulement 'donner un signe' ». La redéfinition du langage en une suite binaire de signes, ou plus exactement de signaux, et donc en des «formules d'un calcul logique ", était donc inscrite comme possibilité dans l'être du langage lui-même. L'histoire du langage pouvait donc maintenant être déchiffrée comme la longue durée de sa déformation en une écriture de calculs - mais comme une déformation due à l'être même du langage. Une fois branchée aux «flux de courant et aux impulsions électriques ", cette évolution menait directement aux grosses machines à calculer de l'époque :

La construction et l'efficacité des machines à calculer géantes reposent sur les principes technico-calculatoires de cette transformation du langage comme dire en un langage comme simple production de signes.

Seul un poème ne pouvait être "en aucun cas programmé ». Cette intuition d'une certaine proximité entre pensée et poésie - les penseurs nommant l'être tandis que les poètes expriment le sacré -, Heidegger la tenait donc en fait tout simplement de ses recherches sur l'origine et les limites de la formalisation ${ }^{68}$. Curieusement, c'est en 1951, à Stuttgart, à l'invitation de Max Bense, l'inventeur de l'esthétique de l'information et grand passionné de cybernétique (c'était dans l'entourage de ce dernier - ce que Heidegger devait bien savoir - qu'avaient lieu depuis la fin des années 50 les essais de production stochastique de textes basés sur des ordinateurs ${ }^{69}$ ), que Heidegger présenta l'expression peut-être la plus hermétique de sa pensée antiformaliste du langage, qu'il voulait laisser pure de tout « avis universellement utilisable sur le langage $\mathrm{e}^{70}$ ».

La question «qu'est-ce que penser?» telle que Heidegger la posait depuis 1950 était parvenue à son expression par le biais d'un éclaircissement historique, mené sur la base de ces modèles de pensée archéologiques très féconds, du processus de formalisation ; or ce processus avait également conduit à l'apparition de la cybernétique. La rencontre 
clarificatrice de ces deux théories était donc prévue de longue date. Elle témoigne d'une époque au seuil d'une coupure épistémique au cours de laquelle à la fois une nouvelle science universelle et son archéologie philosophique firent leur apparition - une période dont nous percevons encore aujourd'hui certaines conséquences directes, au moins en ce qui concerne les problématiques épistémologiques et ontologiques, et ce même si la cybernétique comme évènement historique appartient depuis longtemps au passé.

Ce n'est pas un hasard si Heidegger fit savoir en décembre 1951 à Hannah Arendt : « Je parviens tout juste à proximité des choses dignes d'être pensées ${ }^{71}$ ». Or, la chose la plus digne d'être pensée était la chose de la pensée elle-même. À cette époque, Heidegger s'adressait sans cesse à ses auditeurs en parlant de "nous contemporains». Cela suffisait à signifier le caractère inouï de l'entreprise. La question "Qu'est-ce que penser? » était pour les contemporains la question qu'il fallait encore poser. D’ailleurs, il fallait d'abord "la faire se poser», puisqu'elle attendait encore dans l'ombre de l'« obsolescence » de toute "pensée jusqu'ici pensée », obsolescence qui annonçait une nouvelle « ère ». Et ce alors que « le processus d'accomplissement des temps modernes ne fai [sai]t que commencer ${ }^{72}$ ", au cours duquel la logique occidentale allait devenir la logistique et réaliser son " déploiement irrésistible » vers le « cerveau électronique ${ }^{73}$ ». L'apparition de la question de la pensée constitua l'évènement majeur de cette période de transition, et cette question elle-même était "la question historique ${ }^{74}$ ». La propagation ininterrompue de la théorie de l'information constituait le symptôme le plus visible du fait que la question de l'être de la pensée était encore à venir et constituait la question la plus urgente de l'avenir ${ }^{75}$. Mais quiconque commençait à penser sans partir de l'origine, en s'abstenant d'effectuer l'archéologie, l'analyse de l'origine des machines à calculer et des modélisations cybernétiques du réel, ne pouvait penser les modifications de la pensée qui avaient alors lieu. Heidegger exigeait de ses contemporains qu'ils pensent à la lumière des machines :

Pour la pensée contemporaine, la logique est devenue encore plus logique, ce pourquoi elle s'est donnée le nom dérivé de 'logistique'. Sous ce nom, la logique réalise sa dernière forme de domination, qui est maintenant universelle, planétaire. Cette forme de domination porte à l'ère de la technique les traits d'une machine. Il est bien évident que les machines à calculer qui sont utilisées dans l'économie, dans l'industrie, dans les instituts de recherche scientifiques et dans les centres organisationnels de la politique ne sont pas seulement des outils permettant d'opérer plus rapidement des calculs. La machine à penser est au contraire déjà la conséquence en soi d'une modification de la pensée, qui, en faisant de celle-ci un simple calcul, appelle à sa traduction en la machinerie de ces machines. C'est pourquoi nous passons à côté des transformations de la pensée qui ont lieu sous nos yeux si nous ne percevons pas que la pensée devait devenir logistique dès lors qu'à son origine elle était logique ${ }^{76}$.

Le 30 octobre 1965, Heidegger tint une conférence en l'honneur de Ludwig Binswanger, alors déjà très âgé. À ce moment-là, la lettre devenue célèbre sur la fin de la philosophie par la cybernétique était parvenue depuis plus d'un an à son destinataire, le siège de l'UNESCO à Paris ${ }^{77}$. Ce que Heidegger décrivit lors de l'hommage à Binswanger constituait en de nombreux points une répétition du chef-d'œuvre parisien. Mais Heidegger surprit cependant son auditoire par l'approfondissement d'un point précis : «À la fin de la philosophie, c'est de l'ultime possibilité du mode de penser qui est le sien qu'il commence à s'agir sérieusement ${ }^{78} »$. Comment donc fallait-il comprendre cette expression d'une dernière possibilité de penser philosophiquement? 

"union nouvelle» entre les sciences actuelles et les sciences du passé, chargée de remplacer la philosophie sur le point de se désagréger. Si le «trait caractéristique » de la philosophie depuis l'Antiquité grecque avait consisté à créer, dans l'horizon qu'elle venait d'ouvrir, des sciences qui quant à elles revendiquaient l'auto-suffisance au sein de cet horizon, on assistait maintenant à une évolution, dans la mesure où les sciences désormais «s'affranchiss[ai]ent » de la philosophie ${ }^{79}$. Cette évolution «s'annonce dans le fait que les différents domaines des sciences sont tous conçus en vue d'une action bien spécifique » : celle de la "commande et de l'information». L'union de toutes les sciences réalisée par la cybernétique était cependant « une union en un sens nouveau ", en ce que la cybernétique, délaissant le rayon d'action de la pensée fondatrice qu'était la philosophie, ne constituait plus une "science fondamentale». Ses "conceptions directrices " de la commande et de la rétroaction modifiaient "de façon inquiétante » aux yeux de Heidegger tous les " concepts-clés jusqu'ici canoniques dans les sciences, tels que fondement et conséquence, cause et effet $^{80} »$. Cette modification touchait également et avant tout le principe de tous les principes - le principe de raison suffisante. L'« unité du principe de raison » était abolie, l'unité elle-même devenait un "concept technique au sens le plus strict». La cybernétique était le nom d'un évènement qui ne pouvait plus se penser par la philosophie puisqu'il ne pouvait plus se penser de manière fondamentale, à l'aide de principes. Or, au moment où les catégories abdiquaient au profit de "modèles opératoires » et où, comme l'on sait aujourd'hui, l'ère de la modélisation et de la simulation au sens premier, technologique, débutait, la philosophie était parvenue à la limite de ce qui, à l'aide des moyens offerts jusque-là par la pensée - les seuls moyens dont elle disposait -, était par elle pensable. Elle n'était plus capable de penser l'évènement épistémique de taille qui avait lieu à ce moment précis. Là était sa fin. Elle mourut d'une incapacité à appréhender l'Histoire qui s'écrivait sous ses yeux.

41

en fait l'histoire de la pensée elle-même qui rendit la philosophie obsolète. Alors que depuis ses débuts la philosophie avait eu à penser la présence de ce qui est présent et que le déroulement de son histoire n'avait suivi rien d'autre que les différentes manifestations de cette présence, la dernière manifestation de cette évolution s'était quant à elle délivrée de son emprise. La commande, réalisation extrême des potentialités les plus intimes de la pensée de la représentation, et qui initiait le mouvement vers l'irreprésentable, s'avéra tout simplement impensable. Cela signifia non seulement la fin de la modernité ; cela signifia aussi la fin de la philosophie. Et comme la cybernétique, réalisation extrême de ce qui avait été jusqu'alors, ne pouvait se penser elle-même, elle ne fit qu'indiquer cette fin. D'ailleurs, ce n'est qu'en tant que "signe de la fin de la philosophie » que Heidegger était prêt à lui conférer une quelconque signification philosophique. La «force de l'épuisante sommation » (Macht des herausfordernden Stellens), qui atteignait alors en la cybernétique son point culminant, exigeait au contraire pour pouvoir être caractérisée de manière adéquate « une pensée autre, qui mette en doute l'idée de présence en tant que telle ». Car son apparition apportait avec elle « de l'encore impensé, dont les spécificités ne pouvaient être appréhendées par la pensée philosophique ${ }^{81} »$. Penser « la présence eu égard à ce qui la détermine en tant que telle ", et non seulement appréhender philosophiquement les manifestations de son évolution - telle était la tâche nouvelle qu'avait rendu visible et urgente l'évolution de la situation de l'être causée par la cybernétique. Or, cette tâche exigeait de dépasser la philosophie. "La question de cette définition », Heidegger 
l'indiquait explicitement, «emporte la pensée dans un domaine qui demeure inaccessible à la pensée philosophique ${ }^{82}$. »

Le programme heideggérien et sa relance d'après-guerre avaient donc triomphés. La cybernétique l'avait placé, comme elle avait placé l'histoire, devant une nouvelle tâche de la pensée. En dépit des apparences, cette nouvelle tâche avait l'avantage de mettre, ou plutôt de laisser le destin du monde entre les mains des Européens. Car la logistique et, partant, la cybernétique en tant que sa réalisation étaient selon Heidegger des phénomènes à l'origine européens, fussent-ils au final nous être parvenus après un détour par l'Amérique. Or, de l'origine devait aussi naître la résolution. Dès le séminaire de Zurich de novembre 1951, Heidegger s'était exprimé en ce sens géopolitique sur la situation de la destinée :

Cette conception de la logistique comme la philosophie est liée à l'européanisation. (...) La logistique s'est développée à tel point qu'elle joue maintenant un rôle effrayant dans la recherche mathématique (sur les machines à penser et à calculer), c'est-à-dire que ce qui a commencé avec Descartes s'est développé d'une manière telle qu'en l'état actuel des choses, la Chine aura d'ici quelques décennies, voire quelques siècles, un mode d'existence européen, tout comme le Japon - c'est cela l'européanisation, c'est ce phénomène dans lequel l'être de la pensée moderne est déterminé non seulement par les machines mais aussi par la technique, et dans lequel ce rapport fondamental à l'être détermine l'humanité. Et si je puis formuler mon avis personnel, le retournement de la destinée de l'homme ne pourra probablement partir que du lieu d'où cet état final de notre planète tire son origine $^{83}$.

Impossible par contre de compter sur l'Amérique. Car « ces hommes » - les Américains - n'avaient aucune idée, "à l'instant où l'on parle avec eux de ce qui se passe aujourd'hui », "du lieu où ils se trouvent ${ }^{84}$ ». Les Américains n'éprouvaient aucun " intérêt pour la 'question de l'être'85 », le complexe militaro-industriel les empêchant de percevoir la réalité.

La réalité qu'évoquait Heidegger était marquée par l'ordre du savoir propre à la Guerre froide, dans lequel la cybernétique accomplit le voilement de la question de l'être. En observant l'Amérique, on se retrouvait finalement renvoyé à l'Europe. L'Amérique était la propre question de l'Europe comme figure. L'Amérique était la figure de la question de l'être initialement posée en Europe, et ce dans son oubli le plus abouti. Ce qui était au départ une différence géopolitique revenait sous les traits d'une distinction géoontologique. La question de l'être ne se laissait penser que de manière européenne, que depuis l'Europe, et ce par une descente vers les couches profondes de la tradition européenne.

Peu importe que nous ayons lu ou non ne serait-ce qu'une seule ligne de Heidegger: nous nous trouvons dans l'après de cette pensée qui dévoila face à la cybernétique et dans un vaste programme archéologique le sol technologique sur lequel la pensée toujours se meut et qui clôt à la fois la philosophie et l'oubli de la technique constitutif de cette dernière. Depuis lors, nous ne pensons pas seulement ce qui est, nous ne pensons pas seulement des présences, nous pensons également leur historicité et leur détermination technique et médiale, leur milieu techno-logique. C'est peut-être précisément en cela que nous sommes encore les créatures de l'histoire d'une fascination nommée cybernétique. Nous contemporains, qui voulons être tout, sauf les conteurs de variations parménidiennes. 


\section{BIBLIOGRAPHIE}

Abraham Tara H., « (Physio)Logical circuits: The intellectual origins of the McCulloch-Pitts neural networks ", Journal of the History of Behavioural Sciences, vol; 38, n 1, p. 3-25.

Arendt Hannah, Heidegger Martin, Briefe 1925-1975 und andere Zeugnisse, Francfort, V. Klostermann, 1999.

Arendt Hannah, Heidegger Martin, Lettres de 1925 à 1975 et autres documents, Paris, Gallimard, 2001.

Büscher Barbara, Hermann Hans-Christian von, Hoffmann Christoph (dir.), Ästhetik als Programm. Max Bense / Daten und Streuungen, Berlin, Vice versa, 2004.

Galison Peter, « The Ontology of the Enemy: Norbert Wiener and the Cybernetic Vision », Critical Inquiry, vol. 21, $\mathrm{n}^{\circ} 1,1994$.

Guilbaud Georges-Théodule, « Divagations cybernétiques », in Esprit, septembre 1950, p. 281-295.

Günther Gotthard, « Machine, Seele und Weltgeschichte », in Gotthard Günther, Beiträge zur Grundlegung einer operationsfähigen Dialektik, t. III, Hambourg, Meiner, 1980, p. 211-235.

Günther Gotthard, « Number and Logos. Unforgettable Hours with Warren St. McCulloch » [1975], in Selbstorganisation. Jahrbuch für Komplexität in den Natur-, Sozial - und Geisteswissenschaften 6, 1995, p. 318-348.

Hagen Wolfgang, « Die Kamouflage der Kybernetik », in Claus Pias (dir.), Die Macy-Konferenzen 1946-1953, t. II, Essays \& Dokumente, Zürich/Berlin, 2004, p. 191-207.

Hampe Michael, « Whiteheads Entwicklung einer Theorie der Ausdehnung », in A.N. Whitehead / Helmut Maaßen (dir.), Prozeß, Gefühl und Raum-Zeit. Materialien zu Whiteheads 'Prozeß und Realität' t. I, Francfort, Suhrkamp, 1991, p. 220-243.

Heidegger Martin, « Logos (Heraklit, Fragment 50) », in Martin Heidegger, Vorträge und Aufsätze, Pfullingen, G. Neske, 1954.

Heidegger Martin, « Die Sprache », in Martin Heidegger, Unterwegs zur Sprache, Stuttgart, Neske, 1959, p. 9-33.

Heidegger Martin, Héraclite, Paris, Gallimard, 1973.

Heidegger Martin, « Nachwort zu: "Was ist Metaphysik?" » [1943], in Martin Heidegger, Wegmarken, Francfort, V. Klostermann, 1976.

Heidegger Martin, « La parole », in Martin Heidegger, Acheminement vers la parole, Paris, Gallimard, 1976, p. 11-37.

Heidegger Martin, Sein und Zeit [1927], Gesamtausgabe, t. II, Francfort, 1977.

Heidegger Martin, Metaphysische Anfangsgründe der Logik im Ausgang von Leibniz (cours du semestre d'été 1928), Gesamtausgabe, t. XXVI, Francfort, V. Klostermann, 1978.

Heidegger Martin, « Neuere Forschungen über Logik » [1912], in Martin Heidegger, Frühe Schriften, Gesamtausgabe, t. I, Francfort, V. Klostermann, 1978.

Heidegger Martin, Parmenides (cours du semestre d'hiver 1942/1943), Gesamtausgabe t. 54, Francfort, V. Klostermann, 1982. 
Heidegger Martin, « Die Herkunft der Kunst und die Bestimmung des Denkens » [1967], in Petra Jaeger / Rudolf Lüthe (dir.), Distanz und Nähe. Reflexionen und Analysen zur Kunst und Gegenwart, Würzburg, Königshausen und Neumann, 1983.

Heidegger Martin, « La provenance de l'art et la destination de la pensée », in Michel Haar (dir.), Martin Heidegger, Paris, Éditions de l'Herne, 1983.

Heidegger Martin, Le principe de raison, Paris, Gallimard, 1983.

Heidegger Martin, Qu'appelle-t-on penser?, Paris, PUF, 1983.

Heidegger Martin, « Zeichen », in Martin Heidegger, Aus der Erfahrung des Denkens 1910-1976, Francfort, V. Klostermann, 1983.

Heidegger Martin, Was heißt Denken? (cours de 1951/1952), Tübingen, M. Niemeyer, 1984.

Heidegger Martin, Être et temps, Paris, Gallimard, 1986.

Heidegger Martin, Seminare, Gesamtausgabe, Francfort, t. 15, 1986.

Heidegger Martin, Einführung in die Metaphysik [1935], Tübingen, 1987.

Heidegger Martin, Zollikoner Seminare, M. Boss (ed.), Francfort, 1987.

Heidegger Martin, « Der Anfang des abendländischen Denkens. Heraklit. Sommersemester 1943 », in Martin Heidegger, Heraklit, Gesamtausgabe, t. LV, Francfort, V. Klostermann; 1987.

Heidegger Martin, « Das Ende der Philosophie und die Aufgabe des Denkens » [1965], in Martin Heidegger, Zur Sache des Denkens, Tübingen, M. Niemeyer, 1988, p. 61-80.

Heidegger Martin, Überlieferte Sprache und technische Sprache [1962], Saint-Gallen, Erker, 1989.

Heidegger Martin, « Postface à Qu'est-ce que la métaphysique? ", in Martin Heidegger, Questions I, Paris, Gallimard, 1990.

Heidegger Martin, Langue de tradition et langue technique, Bruxelles, Lebeer-Hossmann, 1990.

Heidegger Martin, «La fin de la philosophie et la tâche de la pensée », in Martin Heidegger, Questions IV, Paris, Gallimard, 1990, p. 279-306.

Heidegger Martin, Introduction à la métaphysique, Paris, Gallimard, 1994.

Heidegger Martin, Der Satz vom Grund, Stuttgart, Neske, 1997.

Heidegger Martin, « Logos (Héraclite, fragment 50) », in Martin Heidegger, Essais et conférences, Paris, 1997, p. 249-278.

Heidegger Martin, Logik als die Frage nach dem Wesen der Sprache (cours du semestre d'été 1934), Gesamtausgabe, t. XXXVIII, Francfort, V. Klostermann, 1998.

Heidegger Martin, « Zur Frage nach der Bestimmung der Sache des Denkens » [1965], in Martin Heidegger, Reden und andere Zeugnisse eines Lebensweges (1910-1976), Gesamtausgabe, t. XVI, Francfort, V. Klostermann, 2000.

Heims Steve Joshua, The Cybernetics Group, Cambridge (Mass.)/Londres, MIT Press, 1991.

Holl Ute, Kino, Trance und Kybernetik, Berlin, Brinkmann U. Bose, 2002.

Hörl Erich, « Parmenideische Variationen. McCulloch, Heidegger und das kybernetische Ende der Philosophie » [« Variations parménidiennes. McCulloch, Heidegger et la fin de la philosophie par la cybernétique »], in Claus Pias (ed.), Cybernetics/Kybernetik. The Macy-Conferences 1946-1953, t. II : Essays und Dokumente, Zurich/Berlin, 2004, p. 209-225. 
Hörl Erich, Die heiligen Kanäle. Über die archaische Illusion der Kommunikation [Les canaux sacrés. Sur l'illusion archaïque de la communication], Zurich/Berlin, Diaphanes Verlag, 2005.

Hörl Erich, « Das kybernetische Bild des Denkens », in Michael Hagner / Erich Hörl (dir.), Die Transformationen des Humanen. Beiträge zurKulturgeschichte der Kybernetik, Francfort, Suhrkamp, 2008.

Husserl Edmund, Gesammelte Schriften, t. III, E. Ströker (ed.), Hambourg, Meiner, 1992. «Expression et signification », in Recherches logiques (trad. fr.), t. II, $1^{\text {re }}$ partie, Paris, 1961, p. 27-123.

Janicaud Dominique, « Heidegger en France », t. II, Entretiens, Paris, A. Michel, 2001.

Lévi-Strauss Claude, La pensée sauvage, Paris, Plon, 1962.

Lowe Victor, Alfred North Whitehead. The Man and his Work, vol. I, 1861-1910, Baltimore/Londres, Johns Hopkins University Press, 1985.

Kay Lily E., « From Logical Neurons to Poetic Embodiments of Mind: Warren S. McCulloch's Project in Neuroscience ", Science in Context, 14/4, 2001, p. 591-614.

Magnus Rudolf, Lane Lectures on Experimental Pharmacology and Medicine, H.H. Dale (ed), Stanford University Publications, University Series, Medical Sciences, vol. 2, nº 3, 1930.

Mauss Marcel, «Rapports réels et pratiques de la psychologie et de la sociologie ", in M. Mauss, Sociologie et anthropologie, Paris, PUF, 1968.

Maxwell James Clerk, « Adress to the Mathematical and Physical Sections of the British Association » [1870], in J.-C. Maxwell, The Scientific Papers, vol. II, W.D. Niven (ed), New York, 1965.

McCulloch Warren, « The brain is a computing machine », Electrical Engineering, 68, 1949, p. $492-497$.

McCulloch Warren, « A Historical Introduction to the Postulational Foundations of Experimental Epistemology » [1964], in W. McCulloch, Embodiments of the Mind, Cambridge (Mass.)/London, 1988, p. 359-372.

McCulloch Warren, Pitts Walter, « A Logical Calculus of the Ideas Immanent in Nervous Activity », in W. McCulloch, Embodiments of the Mind, Cambridge (Mass.)/London, 1988, p. 19-39.

McCulloch Warren, « Through the Den of the Metaphysician » [1948], in W. McCulloch, Embodiments of the Mind, Cambridge (Mass.)/London, 1988, p. 142-156.

McCulloch Warren, « What Is a Number, that a Man May Know It, and a Man, that He May Know a Number? » [1961], in W. McCulloch, Embodiments of the Mind, Cambridge (Mass.)/London, 1988, p. 1-18.

McCulloch Warren, « Why the Mind Is in the Head » [1951], in W. McCulloch, Embodiments of the Mind, Cambridge (Mass.)/London, 1988, p. 72-141.

Mohanty Jitendra Nath, « Heidegger on Logic », Journal of the History of Philosophy, vol. 26, $\mathrm{n}^{\circ} 1$, 1998, p. 107-135.

Most Glenn W., « Zur Archäologie der Archaïk », in Albrecht Dihle et al. (dir.), Antike und Abendland. Beiträge zum Verständnis der Griechen und Römer und ihres Nachlebens, Berlin/New York, 1989, p. 1-23.

Most Glenn W., « Pólemos - Pánton - Patér. Die Vorsokratiker in der Forschung der Zwanziger Jahre », in Hellmut Flashar (dir.), Altertumwissenschaft in den 20er Jahren, Stuttgart, 1995. 
Neumann John von, "The general and logical theory of automata » [1951], in J. von Neumann, Collected Works, vol. V, Abraham H. Taub (ed.), Oxford, Pergamon, 1963, p. 288-328.

Sherrington Charles, Man and his Nature. The Gifford Lectures, Édimbourg, 1937/1938 [1940], Cambridge, 1951.

Smith Roger, « Representations of Mind: C.S. Sherrington and Scientific Opinion, c.1930-1950 », Science in Context, 14/4, 2001, p. 511-539.

Whitehead Alfred North, Process and Reality. An Essay in Cosmology [1929], New York, 1978. Procès et réalité. Essai de cosmologie (trad. fr.), Paris, Gallimard, 1995.

Whitehead Alfred North, The concept of nature, Cambridge, University Press, 1920. Le concept de nature (trad. fr.), Paris, 1998.

Wiener Norbert, « Kybernetik » [1948], in Norbert Wiener, Futurum exactum. Ausgewählte Schriften zur Kybernetik und Kommunikationstheorie, Bernhard Dotzler (ed.), Vienne, 2002.

\section{NOTES}

1. Ce texte est la version augmentée de mon article «Parmenideische Variationen. McCulloch, Heidegger und das kybernetische Ende der Philosophie» [ Variations parménidiennes. McCulloch, Heidegger et la fin de la philosophie par la cybernétique »], publié dans Claus Pias (ed.), Cybernetics/Kybernetik. The Macy-Conferences 1946-1953, t. II : Essays und Dokumente, Zurich/ Berlin, 2004, p. 209-225.

2. Toutes les citations de Maxwell sont tirées de James Clerk Maxwell, «Adress to the Mathematical and Physical Sections of the British Association» (1870), in J.-C. Maxwell, The Scientific Papers, vol. II, éd. par W.D. Niven, New York 1965, p. 215 et sq.

3. Charles Sherrington, Man and his Nature. The Gifford Lectures, Edimbourg, 1937/1938 [1940], Cambridge, 1951, p. 256. Sur le dualisme de Sherrington et la conception de la relation entre le cerveau et l'esprit en vogue entre 1930 et 1950, marquée par un certain conservatisme culturel, cf. Roger Smith: "Representations of Mind: C.S. Sherrington and Scientific Opinion, c. 1930-1950 », in Science in Context, 14/4, 2001, p. 511-539.

4. Warren McCulloch, "Through the Den of the Metaphysician" (1948), in W. McCulloch, Embodiments of the Mind, Cambridge (Mass.)/London, 1988, p. 142-156, p. 143.

5. Ibid., p. 144 (je souligne, E.H).

6. Lily E. Kay, « From Logical Neurons to Poetic Embodiments of Mind: Warren S. McCulloch's Project in Neuroscience », in Science in Context 14/4, 2001, p. 591-614, p. 593.

7. Cf. Warren McCulloch, "A Historical Introduction to the Postulational Foundations of Experimental Epistemology », 1964, in W. McCulloch, Embodiments of the Mind, p. 359-372.

8. Warren McCulloch, Walter Pitts, "A Logical Calculus of the Ideas Immanent in Nervous Activity ", in W. McCulloch, Embodiments of the Mind, p. 19-39, p. 21.

9. Sur le contexte épistémologique précis dans lequel s'inscrivent les calculs de McCulloch et Pitts cf. Kay, "From Logical Neurons... », ainsi que Tara H. Abraham, " (Physio)Logical circuits: The intellectual origins of the McCulloch-Pitts neural networks »; in Journal of the History of Behavioural Sciences, 38, 1, p. 3-25.

10. McCulloch, «Through the Den of the Metaphysician », p. 144.

11. Martin Heidegger, Zollikoner Seminare, M. Boss (ed.), Francfort, 1987, p. 118. Sur le rôle de la guerre dans l'histoire de la cybernétique $c f$. Peter Galison, « The Ontology of the Enemy: Norbert Wiener and the Cybernetic Vision ", in Critical Inquiry, 21, 1 1994, S. 228-266. 
12. Warren McCulloch, "The brain is a computing machine ", in Electrical Engineering, 68 (1949), p. 493.

13. McCulloch plaçait son travail dans la lignée des travaux de Hermann von Helmholtz, de son élève Rudolf Magnus, et surtout de ceux de J.G. Dusser de Barenne, qui influença directement sa conception des fondements de l'épistémologie expérimentale. Rudolf Magnus écrivit par exemple en 1927, dans ses Lane Lectures on Experimental Pharmacology and Medicine qu'il ne put tenir du fait de sa mort soudaine (éd. par H.H. Dale, Stanford University Publications, University Series, Medical Sciences, 1930), sous le titre « The Physiological Apriori » : « L'a priori kantien devait bien sûr être considéré avant tout comme un fait physico-psychologique, comme un facteur déterminant de notre psyche. Il devint cependant vite clair qu'une partie de ces éléments moteurs a priori de notre pouvoir intellectuel avait en fait une base purement physiologique. » (p.97) Selon Magnus, il existe de nombreux mécanismes qui agissent inconsciemment et de manière en partie subcorticale, et qui préparent l'action de notre psyche. Leurs résultats parviennent au cerveau $a$ priori, avant même que ne s'enclenchent la perception sensitive et ses jugements et appréciations psychologiques. McCulloch expliquait en 1961: «La recherche du substrat physiologique de la connaissance se poursuivra tant qu'elle ne sera pas complètement achevée, tant, donc, que nous n'aurons pas obtenu de réponse satisfaisante dans le cadre de la physique, de la chimie, de l'anatomie et de la physiologie du système biologique à la question de savoir comment nous connaissons ce que nous connaissons. » (Warren McCulloch : « What Is a Number, that a Man May Know It, and a Man, that He May Know a Number?", in W. McCulloch, Embodiments of the Mind, 1961, p. 1-18, p. 1). Sur McCulloch et Dusser de Barenne cf. Tara H. Abraham, "(Physio)Logical Circuits ", p. 8 et sqq.

14. McCulloch, «A Logical Calculus of the Ideas Immanent in Nervous Activity », p. 35.

15. McCulloch, «Why the Mind Is in the Head» (1951), in W. McCulloch, Embodiments of the Mind, p. $72-141$, p. 73.

16. Cf. ibid., p. 75-76. McCulloch définit la corruption d'informations comme un rapport entre l' input et l'output: «Nous acquérons notre certitude au prix d'une perte d'information. L'œil transmet au cerveau à peu près un centième de l'information qu'il reçoit. La probabilité pour que ce qu'il transmet soit dû au hasard est ridiculement petite, de l'ordre de $2^{-100}$, un milliardième d'un milliardième d'un milliardième d'un dixième de pourcent. »

17. McCulloch, « What is a Number... », p. 3.

18. McCulloch, "Through the Den of the Metaphysician », p. 144.

19. Cf. Victor Lowe, Alfred North Whitehead. The Man and his Work, vol. I, 1861-1910, Baltimore/ Londres, 1985, p. 136 sqq. (mes remerciements à Michael Hampe).

20. Cf. McCulloch, "Through the Den of the Metaphysician", p.148. Sur la doctrine de l'événement de Whitehead $c f$. Alfred North Whitehead, The concept of nature, Cambridge, 1920, p. 74-98, trad. fr. Le concept de nature, Paris, 1998, p. 89-107.

21. Le fragment 41 dit «La sagesse consiste en une seule chose: savoir qu'une sage raison gouverne tout à travers tout » et le fragment 64 "La foudre gouverne tout » (cités d'après la traduction de Marcel Conche, Paris, 1986, p. 241 et p. 302).

22. Martin Heidegger, Seminare, Gesamtausgabe t. 15, Francfort 1986, p. 32, trad. fr. (modifiée) Héraclite, Paris, 1973, p. 27.

23. Ibid., p. 29, trad. fr. p. 21.

24. Ibid., p. 29, trad. fr. p. 24.

25. Ibid., p. 26, trad. fr. (modifiée) p. 22.

26. Ibid., p. 27, trad. fr. (modifiée) p. 22.

27. McCulloch, «Through the Den of the Metaphysician », p. 144.

28. Cité d'après la traduction de Marcel Conche, Paris : 1996, p.87.

29. Martin Heidegger, Einführung in die Metaphysik (1935), Tübingen, 1987, p.111, trad. fr. (modifiée) Introduction à la métaphysique, Paris, 1994, p. 152. 
30. Martin Heidegger, Was heißt Denken ? (cours de 1951/1952), Tübingen, 1984, p. 148, trad. fr. (modifiée) Qu'appelle-t-on penser?, Paris, 1983, p. 224.

31. Glenn W. Most, « Pólemos - Pánton - Patér. Die Vorsokratiker in der Forschung der Zwanziger Jahre », in Hellmut Flashar (dir.), Altertumwissenschaft in den 20er Jahren, Stuttgart, 1995, p. 89.

32. Marcel Mauss, "Rapports réels et pratiques de la psychologie et de la sociologie", in M. Mauss, Sociologie et anthropologie, Paris, 1968, p. 281-310, p. 309.

33. Cf. notamment Martin Heidegger, Parmenides (cours du semestre d'hiver 1942/1943), Gesamtausgabe t. 54, Francfort, 1982, p. 2.

34. J'ai moi-même tenté de situer plus précisément cette illusion de l'archaïsme dans l'histoire des médias dans mon livre Die heiligen Kanäle. Über die archaische Illusion der Kommunikation [Les canaux sacrés. Sur l'illusion archaïque de la communication], Zurich / Berlin : 2005. Cf. également Glenn W. Most: "Zur Archäologie der Archaïk», in Albrecht Dihle et al. (dir.), Antike und Abendland. Beiträge zum Verständnis der Griechen und Römer und ihres Nachlebens, Berlin/New York, 1989, p. 1-23, ainsi que Ute Holl, Kino, Trance und Kybernetik, Berlin, 2002. Sur le raccourci de LéviStrauss $c f$. notamment La pensée sauvage, Paris, 1962, p. 357.

35. Le mathématicien Georges-Théodule Guilbaud avait dès 1950 vu se développer avec la cybernétique un certain illusionnisme, et l'avait mis en rapport avec la structure de cette nouvelle science : «La confusion n'épargne guère les mots à la mode ; le nom de Cybernétique, en passe de devenir célèbre, est donc en danger. Des associations impropres, des acceptions floues, des mythes même le menacent. Le danger est d'autant plus grand que, par sa nature même, la cybernétique est une science-carrefour: c'est sa raison d'être et son caractère le plus important. » (G.-Th. Guilbaud, «Divagations cybernétiques », in Esprit, septembre 1950, p. 281-295, p. 281).

36. Toutes les citations sont extraites de Gotthard Günther : « Number and Logos. Unforgettable Hours with Warren St. McCulloch» (1975), in Selbstorganisation. Jahrbuch für Komplexität in den Natur-, Sozial - und Geisteswissenschaften 6 (1995), p. 318-348, p. 318 et sqq.

37. Je dois à Martin Hampe cette référence à la distinction, essentielle d'un point de vue historico-épistémologique et géophilosophique, entre ontologie du processus et ontologie de l'objet. Sur l'ontologie du processus $c f$. Alfred North Whitehead, Process and Reality. An Essay in Cosmology (1929), New York, 1978, p. 208 et sqq, trad. fr. Procès et réalité. Essai de cosmologie, Paris, 1995, p. 339 et sqq. Pour une situation plus précise de la doctrine du processus de Whitehead dans l'histoire des sciences, et notamment dans l'histoire des mathématiques, $c f$. Michael Hampe, "Whiteheads Entwicklung einer Theorie der Ausdehnung », in A.N. Whitehead / Helmut Maaßen (dir.), Prozeß, Gefühl und Raum-Zeit. Materialien zu Whiteheads 'Prozeß und Realität' t. I, Francfort, 1991, p. 220-243.

38. Cf. Martin Heidegger, «Die Herkunft der Kunst und die Bestimmung des Denkens » (1967), in Petra Jaeger / Rudolf Lüthe (dir.), Distanz und Nähe. Reflexionen und Analysen zur Kunst und Gegenwart, Würzburg, 1983, p. 11-22, p. 16 et sq., trad. fr. « La provenance de l'art et la destination de la pensée ", in Michel Haar (dir.), Martin Heidegger, Paris, 1983, 1986, p. 84-92, p. 88.

39. McCulloch, «Through the Den of the Metaphysician », p. 156.

40. Cité d'après Steve Joshua Heims, The Cybernetics Group, Cambridge (Mass.), 1991, p. 50 et sq.

41. Norbert Wiener, «Kybernetik» (1948), in Norbert Wiener, Futurum exactum. Ausgewählte Schriften zur Kybernetik und Kommunikationstheorie, Bernhard Dotzler (ed.), Vienne, 2002, p. 15.

42. Sur les réserves, principalement du domaine de la théorie de la mesure, émises par John von Neumann, et qui remettaient en question le statut fondamental de la physiologie du cerveau pour le programme cybernétique et, partant, ce programme lui-même, $c f$. Wolfgang Hagen, «Die Kamouflage der Kybernetik » in Claus Pias (dir.), Die Macy-Konferenzen 1946-1953, t. II, Essays \& Dokumente, Zürich/Berlin, 2004, p. 191-207.

43. John von Neumann, «The general and logical theory of automata » (1951), in J. von Neumann, Collected Works, vol. V, Abraham H. Taub (ed.), Oxford, 1963, p. 288-328, p. 311. 
44. Cf. Gotthard Günther, "Machine, Seele und Weltgeschichte », in Gotthard Günther, Beiträge zur Grundlegung einer operationsfähigen Dialektik, t. III, Hambourg, 1980, p. 211-235, p. 231. J'ai analysé plus en détails ce complexe dans mon article "Das kybernetische Bild des Denkens », in Michael Hagner / Erich Hörl (dir.), Die Transformationen des Humanen. Beiträge zurKulturgeschichte der Kybernetik, Francfort, 2008.

45. Heidegger, Was heißt denken ?, p. 3, trad. fr. p.24.

46. Ibid., p. 156, trad. fr. (modifiée) p. 235.

47. Martin Heidegger, « Der Satz vom Grund » [1956], in Heidegger, Der Satz vom Grund, Stuttgart, 1997, p. 211, trad. fr. (modifiée) Le principe de raison, Paris, 1983, p. 270.

48. Derrida fit cette remarque au cours d'un entretien avec Janicaud. $C f$. Dominique Janicaud, « Heidegger en France », t. II, Entretiens, Paris, 2001, p. 122.

49. Ou bien il en parlait, lorsque cela lui arrivait, dans une langue cryptée et en enfouissant ce passé dans une situation particulière de l'histoire de l'être. Il est pour le moins dérangeant de voir Heidegger traiter pendant de longues pages, dans le cadre de l'exposition de la tâche de la pensée (cours du semestre d'hiver 1951/1952), de l'« esprit de vengeance » de Nietzsche et du "pourchasser» (Nach-stellen) comme dernière forme d'expression de la pensée de la représentation. De toute évidence, Heidegger vit dans son interdiction d'enseigner l'expression d'une volonté de vengeance et se considéra comme la victime des derniers sursauts de la métaphysique de l'ère de la représentation. Or, la pensée de Nietzsche - on ne pouvait être plus explicite envers ceux qui pouvaient entendre - « est consacrée à la délivrance de l'esprit de vengeance. Sa pensée est consacrée à un esprit qui, comme liberté à l'égard de la vengeance, passe avant toute simple fraternisation, mais aussi avant toute volonté de punir purement et simplement, avant tout zèle pour la paix comme avant toute organisation de la guerre, avant l'esprit qui veut fonder et assurer la pax - la paix - par des pactes. L'espace de cette liberté à l'égard de la vengeance se situe aussi bien avant tout pacifisme qu'avant toute politique de violence ; il se situe avant toute faiblesse et tout laisser-aller, avant la lâcheté devant le sacrifice, aussi bien qu'avant l'aveuglement de l'action à tout prix. » (Was heißt denken ?, op. cit. p. 33, trad. fr. p. 65)

50. Ibid., p. 10, trad. fr. p. 34 .

51. Ibid., p. 102., trad. fr. (modifiée) p. 160.

52. Ibid., p. 5, trad. fr. (modifiée) p. 27.

53. Martin Heidegger, « Neuere Forschungen über Logik» [1912], in Heidegger, Frühe Schriften, Gesamtausgabe, t. I, Francfort, 1978, p. 18.

54. Cf. la première Recherche logique " Ausdruck und Bedeutung ", in Edmund Husserl, Gesammelte Schriften, t. III, E. Ströker (ed.), Hambourg, 1992, p. 30-110, et notamment p. 72 et sq. (trad. fr. «Expression et signification », in Recherches logiques, t. II, $1^{\text {re }}$ partie, Paris, 1961, p. 27-123, p. 78 et sq.)

55. Martin Heidegger, Sein und Zeit [1927], Gesamtausgabe, t. II, Francfort, 1977, p. 196, trad. fr. Être et temps, Paris, 1986, p. 192. Cf. également J.N. Mohanty, « Heidegger on Logic », in Journal of the History of Philosophy, 26, 1, 1998, p. 107-135. Mohanty pense lui aussi que ce sont ses recherches sur le problème du jugement et donc son questionnement sur la logique qui conduisirent Heidegger à l'ontologie et à la métaphysique.

56. Heidegger, Sein und Zeit, p. 205, trad. fr. p. 200.

57. Ibid., p. 212, trad. fr. p. 206.

58. Martin Heidegger, Metaphysische Anfangsgründe der Logik im Ausgang von Leibniz, cours du semestre d'été 1928, Gesamtausgabe, t. XXVI, Francfort, 1978, p. 27.

59. Ibid., p.127.

60. Ibid., p. 59.

61. Martin Heidegger, Einführung in die Metaphysik, p. 89, trad. fr. (modifiée) p. 124-125. Il faut cependant noter que le néologisme heideggérien d'une "pensée de la représentation" 
(Vorstellungsdenken) porte la trace de son origine, qui remonte à la critique que le 19ème siècle finissant fit du psychologisme. L'éducation philosophique de Heidegger avait été marquée par l'opposition frontale à la représentation, c'est-à-dire par le refus de la représentation comme unité logique. Par son concept d'une "pensée de la représentation », il parvint à renverser le problème de la représentation pour en faire le symbole de l'époque, et à transformer le problème gnoséologique en un problème historico-ontologique.

62. Martin Heidegger, «Logos (Heraklit, Fragment 50)», in Heidegger, Vorträge und Aufsätze, Stuttgart: 1954, p .229, trad. fr. "Logos (Héraclite, fragment 50)», in Heidegger, Essais et conférences, Paris, 1997, p. 249-278, p. 278.

63. Martin Heidegger, «Der Anfang des abendländischen Denkens. Heraklit. Sommersemester 1943 », in Heidegger, Heraklit, Gesamtausgabe, t. LV, Francfort, 1987, p. 137.

64. Martin Heidegger, «Grundsätze des Denkens », Freiburger Vorträge 1957, Gesamtausgabe t. LXXIX, Francfort, 1994, p. 161.

65. Martin Heidegger, Logik als die Frage nach dem Wesen der Sprache, cours du semestre d'été 1934, Gesamtausgabe, t. XXXVIII, Francfort, 1998, p. 8.

66. Martin Heidegger, «Zeichen », in Heidegger, Aus der Erfahrung des Denkens 1910-1976, Francfort, 1983, 2002, p. 211.

67. Cette citation ainsi que les suivantes sont extraites de Martin Heidegger, Überlieferte Sprache und technische Sprache [1962], Saint-Gallen, 1989, p. 22 et sqq, trad. fr. (en partie modifiée) Langue de tradition et langue technique, Bruxelles, 1990, p. 17 et sqq.

68. Martin Heidegger, « Nachwort zu: "Was ist Metaphysik ?" » (1943), in Heidegger, Wegmarken, Francfort, 1976, p. 309, trad. fr. "Postface à Qu'est-ce que la métaphysique?", in Heidegger, Questions I, Paris, 1990, p. 73-84, p. 80-81.

69. Cf. à ce sujet Barbara Büscher, Hans-Christian von Hermann, Christoph Hoffmann (dir.), Ästhetik als Programm. Max Bense / Daten und Streuungen, Berlin, 2004, p. 154-225.

70. Heidegger, «Die Sprache ", in Heidegger, Unterwegs zur Sprache, Stuttgart, 1959, p. 9-33, p. 12, trad. fr. « La parole », in Heidegger, Acheminement vers la parole, Paris, 1976, p. 11-37, p. 14.

71. Hannah Arendt, Martin Heidegger, Briefe 1925-1975 und andere Zeugnisse, Francfort, 1999, p. 132, trad. fr. (modifiée), Hannah Arendt, Martin Heidegger, Lettres de 1925 à 1975 et autres documents, Paris, 2001, p. 131.

72. Heidegger, Was heißt denken?, p. 161, trad. fr. p. 243.

73. Ibid., p. 145, trad. fr. p. 220.

74. Ibid., p. 103, trad. fr. (modifiée) p. 161.

75. C'est ainsi que Heidegger avait prévu de conclure son cours, qu'il n'eut pas le temps de terminer, par ces mots : «La volonté croissante de 'transformer' la doctrine traditionnelle de la pensée - la logique - en de la logistique, le fait que l'on mise de plus en plus sur la transformation du langage dans son ensemble en de la technique logistique [...] sont des signes indiquant que l'accomplissement de la métaphysique, qui vit de l'ignorance de la dualité de son origine essentielle, n'en est qu'à ses premiers tâtonnements.» (Heidegger, Was heißt denken?, Gesamtausgabe t. VIII, Francfort : 2002, p. 265)

76. Martin Heidegger, « Grundsätze des Denkens », p. 104 et sq.

77. Cf. Martin Heidegger, « Das Ende der Philosophie und die Aufgabe des Denkens » [1965], in Heidegger, Zur Sache des Denkens, Tübingen, 1988, p. 61-80, trad. fr. « La fin de la philosophie et la tâche de la pensée ", in Heidegger, Questions IV, Paris, 1990, p. 279-306.

78. Martin Heidegger, «Zur Frage nach der Bestimmung der Sache des Denkens » [1965], in Heidegger, Reden und andere Zeugnisse eines Lebensweges (1910-1976), Gesamtausgabe, t. XVI, Francfort, 2000, p. 621, trad. fr. L'affaire de la pensée (Pour aborder la question de sa détermination), Mauvezin, 1990, p. 15.

79. Cf. Heidegger, «Das Ende der Philosophie und die Aufgabe des Denkens », p.63, trad. fr. p. 284. 
80. Toutes les citations sont tirées de Heidegger, «Zur Frage nach der Bestimmung der Sache des Denkens », p. 620, trad. fr. (modifiée) p. 15-16.

81. Ibid., p. 628, trad. fr. (modifiée) p. 23.

82. Ibid., p. 627 et sq., trad. fr. (en partie modifiée) p. 22.

83. Heidegger, Seminare, p. 437 et sq.

84. Ibid.

85. Ibid., p. 359.

\section{AUTEUR}

\section{ERICH HÖRL}

Professeur de Philosophie des Médias et de Technologie des Médias à la Ruhr-Université de Bochum (Allemagne) 